\title{
Elderly Male with Retroperitoneal Myxoid Liposarcoma: An Unsual Presentation- A Case Report
}

Dr. Sarita Nibhoria MD ${ }^{1}$, Dr. Ekta Rani MD², Dr. Amandeep Kaur MBBS ${ }^{3}$, Dr. Shilpa MBBS ${ }^{4}$, Dr. Navjot Kaur MBBS

${ }^{1}$ Professor, ${ }^{2}$ Assistant Professor, ${ }^{3-5}$ Junior Resident, Department of Pathology, GGS Medical College, Faridkot, Punjab, India

DOI: $10.36347 /$ sjmcr.2021.v09i04.025

| Received: 24.02.2021 | Accepted: 10.04.2021 | Published: 26.04.2021

*Corresponding author: Dr. Ekta Rani

Abstract

Case Report

Retroperitoneal sarcomas are rare neoplasms that account for only $1 \%-2 \%$ of all solid tumors and 5\% of primary retroperitoneal liposarcomas are reported as myxoid or round cell liposarcoma [1]. We report the case of a 60-year-old male with a retroperitoneal myxoid liposarcoma of $22 \times 15 \times 8 \mathrm{~cm}$ in size. The only symptoms were persistent pain and progressive tenderness of the abdomen lasting for two months. The mass was radically excised during laparotomy. CT and MRI were useful to clarify the site of origin of the tumor but final diagnosis was based on histological findings.

Keywords: Retroperitoneal sarcomas, round cell liposarcoma, CT and MRI, tumor.

Copyright $(\odot 2021$ The Author(s): This is an open-access article distributed under the terms of the Creative Commons Attribution 4.0 International License (CC BY-NC 4.0) which permits unrestricted use, distribution, and reproduction in any medium for non-commercial use provided the original author and source are credited.

\section{INTRODUCTION}

According to the World Health Organization (WHO), soft-tissue liposarcomas are categorized into five distinct histological subtypes: well-differentiated, dedifferentiated, myxoid, pleomorphic and mixed type.

Liposarcoma is one of the most frequent soft tissue sarcomas found in adults. Most of sarcomas occur outside the retroperitoneum. The main site of origin is the thigh $(13-60 \%)$ while retroperitoneum is involved in 10-36\% of cases [1-3]. Retroperitoneal sarcomas are rare tumors that account for only $1 \%-2 \%$ of all solid tumor. Retroperitoneal sarcomas are malignant tumors arising from mesenchymal cells, which are usually located in muscle, fat, and connective tissues.

Almost all primary retroperitoneal liposarcomas can be classified as well-/dedifferentiated liposarcoma. Rarely, however, primary retroperitoneal liposarcoma is classified as myxoid/round cell liposarcoma, based on the presence of myxoid areas and vascular crow's feet pattern. Myxoid Liposarcoma occurs predominantly in the extremities of young adults and has a disproportionately high tendency to metastasize to unusual soft tissue locations, before giving pulmonary metastasis or disseminated spread. We report a rare case of retroperitoneal myxoid liposarcoma in a 60 year old male.

\section{Case Report}

A 60-year old male presented with complaints of painless distension of abdomen for the last 1 year, which was insidious in onset and gradually progressive in nature. There was history of weight loss for the past 6 months. There was no history of hemoptysis, malena, and bleeding per rectum. Past history was not suggestive of any chronic illness. On physical examination patient was: afebrile; pulse: 90/min and regular; blood pressure-126/80 $\mathrm{mm} \mathrm{Hg}$.

On per abdomen examination: abdomen was distended; flanks were full, soft, and nontender. A palpable retroperitoneal lump occupying left hypochondrium, left lumbar, and left iliac fossa, involving umbilical region and crossing midline was felt with no evidence of ascitis. Rest of systemic examination was within normal limits. Abdominal ultrasonography showed a solid, heterogeneous mass with multiple foci of variable size, probably starting from retroperitoneum, occupying most of abdominal cavity. Computed tomography (CT) of the abdomen and pelvis confirmed the presence of a $22 \times 15 \times 8 \mathrm{~cm}$ retroperitoneal mass.

At laparotomy, a huge mass was found, completely excised and sent to pathology department of GGSMCH, Faridkot for histopathological examination. 
Grossly, the mass was well encapsulated and multilobed with gelatinous consistency and was received in multiple pieces collectively measuring $22 \times 15 \times 8 \mathrm{~cm}$ (Figure-1).

Microscopically: Sections examined show a tumor comprising of round to polyhedral cells, having eccentric nuclei and abundant eosinophilic granular and microvacuolated cytoplasm, with prominent myxoid areas. Prominent vascularity in the form of chicken wire capillary network also noted, suggesting a histological diagnosis of a retroperitoneal Myxoid Liposarcoma. (Figure-2) which was confirmed on Immunohistochemistry staining. IHC showed S-100 positivity.

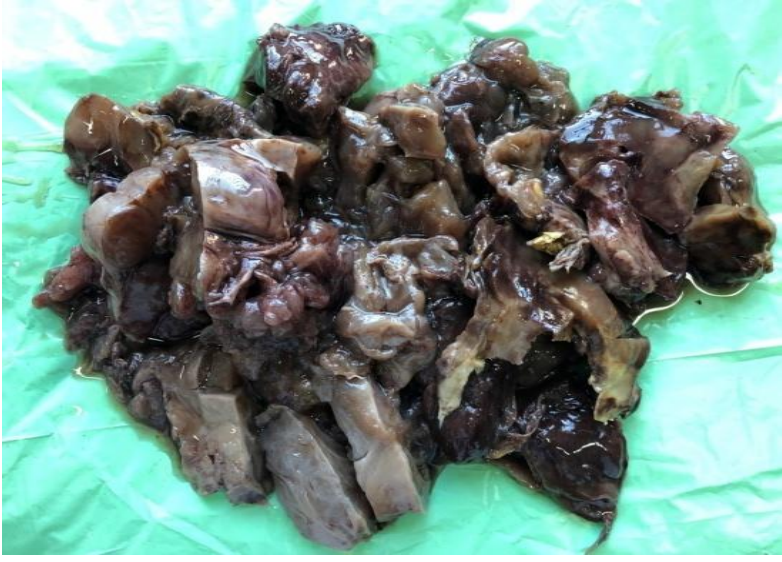

Fig-1: Gross specimen of Resected tumor mass

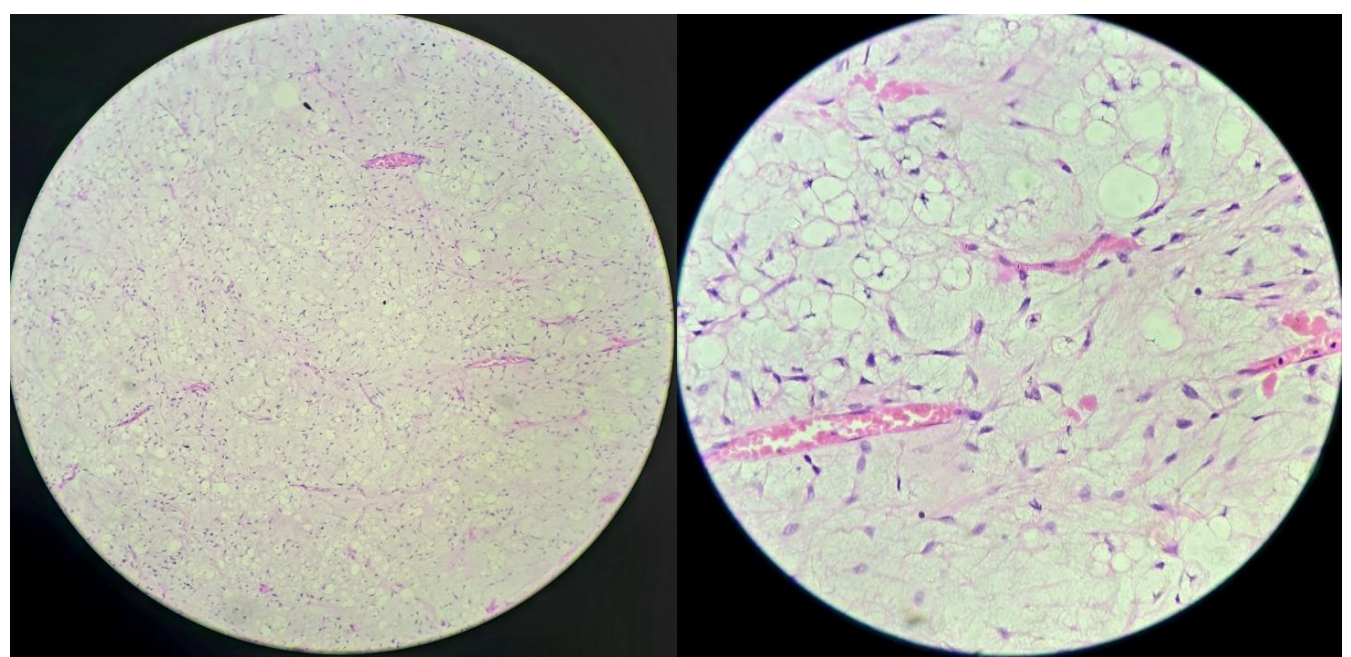

Fig-2: Microscopic examination- myxoid stroma and capillary (10X view, 40X view)

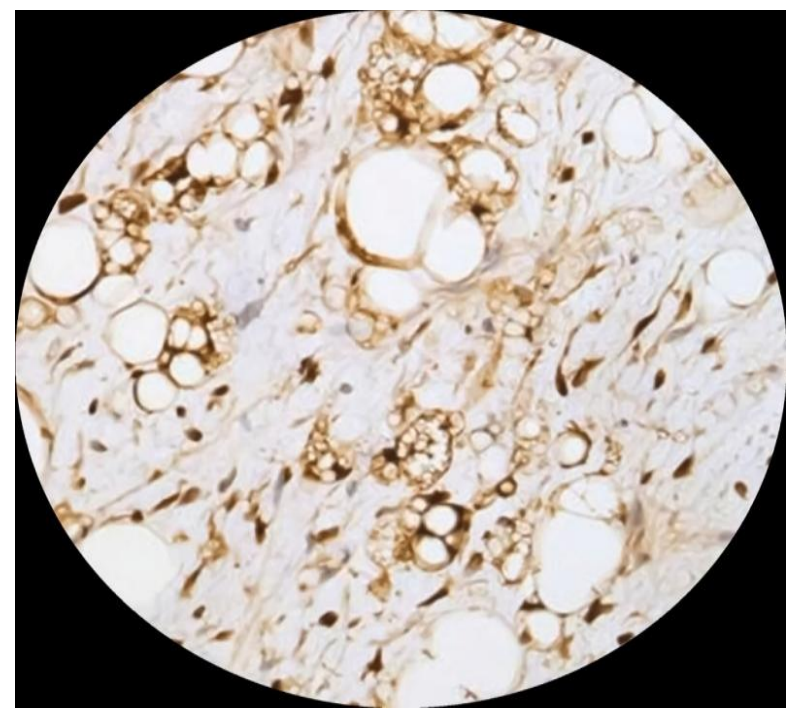

Myxoid Liposarcoma- Immunohistochemical reactivity for S-100 at 40X view

\section{DiscuSSION}

Retroperitoneal sarcomas are rare tumors that account for only $1 \%-2 \%$ of all solid tumors. Most of sarcomas occur outside of the retroperitoneum and only $10 \%-20 \%$ of them originate in the retroperitoneal space. Liposarcoma is the most frequent histological type of retroperitoneal sarcoma, corresponding to $41 \%$ of these tumors [4]. Liposarcomas are subdivided into four well recognized subgroups based on morphology and cytogenic abnormalities: well differentiated, dedifferentiated, myxoid/round cell, and pleomorphic [5]. Liposarcoma occurs most commonly in the extremities (52\%), retroperitoneum (19\%), and inguinal region $(12 \%)$ [6]. On the basis of histological appearance with myxoid areas and vascular crow's feet pattern, $5 \%$ of primary retroperitoneal liposarcomas are reported as myxoid or round cell liposarcoma [5]. It has been reported that $20 \%$ of the tumors are $>10 \mathrm{~cm}$ at the time of presentation [7]. These tumors of mesodermal origin are known to reach significant dimensions, despite their poor vascularization and can grow to enormous size, weighing over 100 pounds. The prognosis varies depending on the site of origin, the type of cancer cell, the tumor size, the depth, and proximity to lymph nodes. Well-differentiated liposarcomas and myxoidliposarcomas have a good prognosis and their rates of metastasis are low compared to the other types. Apart from different histology and malignancy, myxoid/round-cell LS and well-differentiated/dedifferentiated LS are different entities; in more than $95 \%$ of myxoid/round-cell LS, a 
classical $\mathrm{t}(12 ; 16)(\mathrm{q} 13 ; \mathrm{p} 11)$ or $\mathrm{t}(12 ; 22)(\mathrm{q} 13 ; \mathrm{q} 12)$ translocation can be found, which results in fusion of FUS-CHOP or EWSR1-CHOP gene, respectively. Characteristic genetic alteration for welldifferentiated/dedifferentiated LS is the amplification of the 12q13-15 region, which includes MDM2 and CDK4 genes [8].

Complete surgical excision is the mainstay of treatment for retroperitoneal sarcomas with emphasis to obtain negative microscopic margins.

Hatano et al., describe that if a myxoid LS is adjacent to critical structures and wide resection cannot be performed, marginal or intralesional resection combined with postoperative radiotherapy (50-70 Gy, average $59.2 \mathrm{~Gy}$ ) can achieve a high rate of local control [9].

\section{CONCLUSION}

Retroperitoneal sarcomas are rare tumors, only $5 \%$ of primary retroperitoneal liposarcomas are reported as myxoid or round cell liposarcoma. Histopathology is the gold standard for the diagnosis and Immunohistochemistry aids in the final diagnosis.

\section{REFERENCES}

1. McGrath PC. Retroperitoneal sarcomas, Seminars in Surgical Oncology. 1994;10(5): 364-68.

2. Mettlin C, Priore R, Rao U, Gamble D, Lane W, Murphy GP. Results of the national soft- tissue sarcoma registry. Journal of surgical oncology. 1982 Apr;19(4):224-7.
3. Dalal KM, Kattan MW, Antonescu CR, Brennan MF, Singer S. Subtype specific prognostic nomogram for patients with primary liposarcoma of the retroperitoneum, extremity, or trunk. Annals of surgery. 2006 Sep;244(3):381-89.

4. Herrera-Gómez Á, Ortega-Gutiérrez C, Betancourt AM, Luna-Ortiz K. Giant retroperitoneal liposarcoma. World Journal of Surgical Oncology. 2008 Dec;6(1):1-6.

5. Singer S, Antonescu CR, Riedel E, Brennan MF. Histologic subtype and margin of resection predict pattern of recurrence and survival for retroperitoneal liposarcoma. Annals of surgery. 2003 Sep;238(3):358-71.

6. Russell WO, Cohen J, Enzinger F, Hajdu SI, Heise H, Martin RG, Meissner W, Miller WT, Schmitz RL, Suit HD. A clinical and pathological staging system for soft tissue sarcomas. Cancer. 1977 Oct;40(4):1562-70.

7. Inoue $\mathrm{K}$, Higaki $\mathrm{Y}$, Yoshida $\mathrm{H}$. Giant retroperitoneal liposarcoma. International Journal of Urology. 2005 Feb;12(2):220-2.

8. De Vreeze RS, De Jong D, Tielen IH, Ruijter HJ, Nederlof PM, Haas RL, Van Coevorden F. Primary retroperitoneal myxoid/round cell liposarcoma is a nonexisting disease: an immunohistochemical and molecular biological analysis. Modern Pathology. 2009 Feb;22(2):22331.

9. Hatano H, Ogose A, Hotta T, Kawashima H, Sugita T, Sasamoto R, Endo N. Treatment of myxoid liposarcoma by marginal or intralesional resection combined with radiotherapy. Anticancer research. 2003 May 1;23(3C):3045-9. 